\title{
Zika Virus Proteins NS2A and NS4A Are Major Antagonists that Reduce IFN- $\beta$ Promoter Activity Induced by the MDA5/RIG-I Signaling Pathway
}

\author{
Nguyen Thi Thuy Ngan ${ }^{1}$, Seong-Jun $\mathrm{Kim}^{2}$, Jeong Yoon Lee ${ }^{1 *}$, and Jinjong Myoung ${ }^{1 *}$ \\ ${ }^{1}$ Korea Zoonosis Research Institute, Department of Bioactive Material Science and Genetic Engineering Research Institute, Chonbuk National \\ University, Jeonju 54531, Republic of Korea \\ ${ }^{2}$ Center for Convergent Research of Emerging Virus Infection, Korea Research Institute of Chemical Technology, Daejeon 34114, Republic of Korea
}

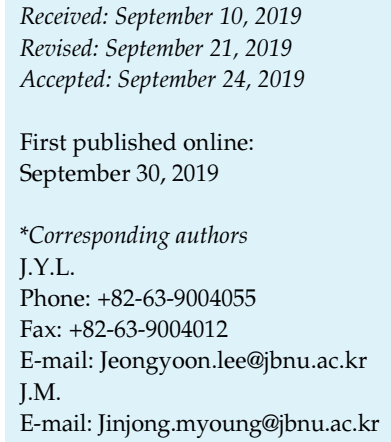

Zika virus (ZIKV) is a mosquito-transmitted, emerging Flavivirus that causes Guillain-Barré syndrome and microcephaly in adults and fetuses, respectively. Since ZIKV was first isolated in 1947, severe outbreaks have occurred at various places worldwide, including Yap Island in 2007, French Polynesia in 2013, and Brazil in 2015. Although incidences of ZIKV infection and dissemination have drastically increased, the mechanisms underlying the pathogenesis of ZIKV have not been sufficiently studied. In addition, despite extensive research, the exact roles of individual ZIKV genes in the viral evasion of the host innate immune responses remain elusive. Besides, it is still possible that more than one ZIKV-encoded protein may negatively affect type I interferon (IFN) induction. Hence, in this study, we aimed to determine the modulations of the IFN promoter activity, induced by the MDA5/RIG-I signaling pathway, by over-expressing individual ZIKV genes. Our results show that two nonstructural proteins, NS2A and NS4A, significantly down-regulated the promoter activity of IFN- $\beta$ by inhibiting multiple signaling molecules involved in the activation of IFN- $\beta$. Interestingly, while NS2A suppressed both full-length and constitutively active RIG-I, NS4A had inhibitory activity only on full-length RIG-I. In addition, while NS2A inhibited all forms of IRF3 (full-length, regulatory domain-deficient, and constitutively active), NS4A could not inhibit constitutively active IRF3-5D. Taken together, our results showed that NS2A and NS4A play major roles as antagonists of MDA5/RIG-I-mediated IFN- $\beta$ induction and more importantly, these two viral proteins seem to inhibit induction of the type I IFN responses in differential mechanisms. We believe this study expands our understanding regarding the mechanisms via which ZIKV controls the innate immune responses in cells and may pave the way to development of ZIKV-specific therapeutics.

Keywords: Zika virus, interferon, evasion

\section{Introduction}

Zika virus (ZIKV) was first isolated from a febrile sentinel rhesus monkey (Rhesus 766) of the Zika Forest in Uganda in 1947 [1]. The first serious outbreak in Yap Island in 2007 was followed by the 2013 outbreak in French Polynesia and the outbreaks throughout Central and South America and the Caribbean regions [2-4]. ZIKV commonly causes mild symptoms such as rash, fever, arthralgia and conjunctivitis; however, the outbreak in Brazil warned that ZIKV infection can be associated with microcephaly in fetuses and the Guillain-Barre syndrome in adults [5-8]. ZIKV is mainly transmitted to humans by two Aedes mosquitoes, Aedes aegypti and Aedes albopictus [9]. New transmission routes of ZIKV have recently been determined in several cases, for example, from mother to fetus during pregnancy and 
breastfeeding after birth, and from one human to another via sexual intercourse or blood transfusion [10].

ZIKV is a single-stranded, positive sense RNA virus belonging to the genus Flavivirus of the family Flaviviridae [9]. The 11-kb genome of ZIKV encodes three structural proteins: the capsid $(\mathrm{C})$, precursor membrane protein (prM), and envelope (E), and seven nonstructural proteins: NS1, NS2A, NS2B, NS3, NS4A, NS4B, and NS5.

Interferon (IFN) production is an important element of the host immune response against viral infection and replication [11, 12]. The viral genome, especially of RNA viruses, is recognized by two pattern recognition receptors (PRRs) named 'melanoma differentiation-associated gene 5' (MDA5) and 'retinoic acid-inducible gene 1' (RIG-I). Recognition of cognate ligands by and activation of PRRs lead to expression of type I IFNs $[12,13]$ and subsequently induce the production of pro-inflammatory cytokines by stimulating nuclear factor kappa-light-chain-enhancer of activated B cells (NF- $\mathrm{B}$ ) [14]. The activation of the downstream signaling molecules of the MDA5/RIG-I pathway, including the mitochondrial antiviral adaptor protein (MAVS), inhibitor of kappa-B kinase epsilon (IKK $\varepsilon$ ), TANK-binding kinase 1 (TBK1), and interferon regulatory factor 3 (IRF3), plays a crucial role in modulating the expression of type I IFN genes. Type 1 IFNs in turn activate the IFN-stimulated response element (ISRE) in an autocrine and paracrine manner and initiate a cascade of signaling events leading to IFN-stimulated genes (ISGs), which function to hinder virus replication.

In this study, we demonstrate that the ZIKV NS2A and NS4A proteins play major roles in antagonizing IFN- $\beta$ production. NS2A and NS4A suppressed IFN- $\beta$ promoter activities through not only down-regulation of RIG-I-like receptors (RLRs) but also the downstream components of the MDA5/RIG-I signaling pathway. Furthermore, ZIKV NS1 also contributed to moderate reduction in IFN- $\beta$ levels by down-regulation of MDA5, constitutively active RIG-I (RIG-I-1-228), MAVS, TBK1, and IKK $\varepsilon$ although the effect was not as strong as those of NS2A or NS4A. Better understanding of the strategies used by ZIKV to overcome the host innate immunity will pave the way to development of antivirals and preventive vaccines.

\section{Materials and Methods}

\section{Cells}

Human embryonic kidney 293T (HEK293T) cells and Vero-E6 cells were obtained from the American Type Culture Collection (ATCC) and maintained in high-glucose $(4,500 \mathrm{mg} / \mathrm{ml})$ Dulbecco's modified Eagle's medium (DMEM) (Welgene, Korea) supplemented with $10 \%$ fetal bovine serum (FBS) (Welgene) and 1\% penicillin/ streptomycin (Gibco, USA) [15-17]. The cells were incubated at $37^{\circ} \mathrm{C}$ in a $5 \% \mathrm{CO}_{2}$ humidifying incubator. Trypsin-EDTA $(0.05 \%)$ and Dulbecco's phosphate-buffered saline (DPBS) were also purchased from Welgene.

\section{Antibodies [18-22].}

A mouse monoclonal anti-FLAG antibody (M2) (1:5000) was purchased from Millipore Sigma, USA. HA-tagged (6E2) mouse monoclonal antibody (1:1000), horseradish peroxidase (HRP)conjugated rabbit monoclonal anti-GAPDH (14C10) (1:2000), HRP-conjugated anti-rabbit $\operatorname{IgG}$ antibody (1:2000) and HRPconjugated anti-mouse IgG antibody (1:2000) were purchased from Cell Signaling, USA.

\section{Reagents}

Opti-MEM Minimal Essential Medium (MEM) (Gibco), polyethylenimine (PEI; Millipore Sigma) and cOmplete Mini Protease Inhibitor Cocktail (Millipore Sigma) were purchased and used for plasmid transfection. The luciferase assay system and Beta-Glo Assay System were obtained from Promega, USA. $4 \times$ Laemmli sample buffer and 2-mercatoethanol were purchased from Bio-Rad, USA. Amersham ECL Prime western blotting detection reagent, Amersham ECL western blotting detection reagent, and Amersham Protran 0.45 nitrocellulose (NC) blotting membrane were purchased from GE Healthcare Life Sciences, USA. EcoRI-HF, BamHI-HF, NotI-HF, NheI-HF, PmeI, PmlI, XhoI, Xbal, and T4 DNA ligase were purchased from New England Biolabs (NEB), USA. MAX Efficiency DH5a competent cells were purchased from Thermo Fisher Scientific, USA. Pfu Plus DNA polymerase was purchased from Elpisbio, Republic of Korea.

\section{Plasmids}

Generation of the pcDNA3.1-Neo-JY3 vector has been described previously [23]. The pcDNA3.1-Neo-JY3-3× FLAG-N vector was modified from pcDNA3.1-Neo-JY3. Briefly, the pcDNA3.1-NeoJY3 vector was digested using NheI-HF and PmeI. The linearized vector was ligated with a linker containing a $3 \times$ FLAG N-terminus tag (5'-GCTAGCGCCACCATGGACTACAAGGACCACGACGGT GACTACAAGGACCACGACATCGACTACAAGGACGACGAC GACAAGCTTATCGATATCACCGGTACCTCAGGATCCTGCA GGCCGGCCCGTACGCGGCCGCTCGAGGTTAACTCTAGAGG GCCCGTTTAAAC-3'). ZIKV RNA was extracted from the supernatant of ZIKV-infected Vero-E6 cells using TRiZol LS (Thermo Fisher Scientific) following the manufacturer's instructions. Reverse transcription (RT) was performed using a primer targeting the 3'-end of the ZIKV genome. Each ZIKV gene was amplified using Pfu Plus DNA polymerase (Elpisbio) under the following conditions: $95^{\circ} \mathrm{C}, 20 \mathrm{~s} ; 58^{\circ} \mathrm{C}, 20 \mathrm{~s} ; 72^{\circ} \mathrm{C}, 10 \mathrm{~s} / \mathrm{kb}$ for 30 cycles. The amplicons were purified using the FavorPrep GEL/PCR Purification Kit (Favorgen, Taiwan) according to the manufacturer's instructions. All the primers used are listed in Table 1. Linearized vectors were 
Table 1. Primers for cloning of ZIKV genes.

\begin{tabular}{ll}
\hline \multicolumn{1}{c}{ Name } & \multicolumn{1}{c}{ Sequence $\left(5^{\prime}-\mathbf{3}^{\prime}\right)$} \\
\hline ZIKV-C-F & CCGGAATTCAAAAACCCAAAAAAGAATCCGGAGG \\
ZIKV-C-R & CCGCTCGAGTTATCGTCTCTTCTTCTCCTTCCTAGC \\
ZIKV-prM-F & CCGGAATTCGCGGAGGTCACTAGACGTGGG \\
ZIKV-prM-R & CCGCTCGAGTTAGCTGTATGCCGGGGCAATCAG \\
ZIKV-E-F & CCGGAATTCATCAGGTGCATAGGAGTCAGCAATAG \\
ZIKV-E-R & CCGCTCGAGTTAAGCAGAGACGGCTGTGGATAAG \\
ZIKV-NS1-F & CCGGAATTCATCAGGTGCATAGGAGTCAGCAATAG \\
ZIKV-NS1-R & CGCTCGAGTTATGCAGTCACCATTGACCTTACTAAGTTGC \\
ZIKV-NS2A-F & CCGGAATTCGGATCAACTGATCACATGGACCACTTC \\
ZIKV-NS2A-R & CCGCTCGAGTTACCGCTTCCCACTCCTTGTGAGC \\
ZIKV-NS2B-F & CCGGAATTCAGCTGGCCCCCTAGCGAAGTAC \\
ZIKV-NS2B-R & CCGCTCGAGTTACCTTTTTCCAGTCTTCACGTATACGTACC \\
ZIKV-NS3-F & CCGGAATTCAGTGGTGCTCTATGGGATGTGCC \\
ZIKV-NS3-R & CCGCTCGAGTTATCTTTTCCCAGCGGCAAACTCCTTG \\
ZIKV-NS4A-F & CCGGAATTCGGAGCGGCTTTTGGAGTGATGG \\
ZIKV-NS4A-R & CCGCTCGAGTTATCTTTGCTTTTCTGGCTCAGGTATGAGC \\
ZIKV-NS4B-F & CCGGAATTCAATGAACTCGGATGGTTGGAGAGAAC \\
ZIKV-NS4B-R & CCGCTCGAGTTAACGTCTCTTGACCAAGCCAGC \\
ZIKV-NS5-F & CCGGAATTCGGGGGTGGAACAGGAGAGACC \\
ZIKV-NS5-R & CCGCTCGAGTTACAGCACTCCAGGTGTAGACCCTTC \\
\hline
\end{tabular}

dephosphorylated using calf intestinal alkaline phosphatase (CIP; $\mathrm{NEB})$ at $37^{\circ} \mathrm{C}$ for $60 \mathrm{~min}$. The ligated products were transformed into DH5 $\alpha$ cells. The constructions of the pcDNA3.1-Hygro-JY4HAN-GS3 vector and plasmids harboring genes involved in the type I interferon pathway, such as MDA5, RIG-I, MAVS, IKKE, TBK1, and IRF3 have been described previously [24, 25]. RIG-I-1228 and IRF3-1-390 were cloned into pcDNA3.1-Hygro-JY4-HANGS3 using conventional cloning methods. In addition, a part of IRF3 (Table 2) was synthesized at Bionics (Republic of Korea) to generate a constitutive active form named 'IRF3-5D' (S396D, S398D, S402D, T404D, and S405D). The synthesized IRF3-5D fragment was replaced with the corresponding region of the pcDNA3.1Hygro-JY4-HAN-GS3-IRF3 construct using PmlI and XhoI (NEB).

\section{Transfection and Luciferase Assay}

HEK293T cells $\left(8 \times 10^{5}\right)$ per well were seeded on a 6-well plate
$24 \mathrm{~h}$ prior to transfection [26]. A mixture containing $500 \mathrm{ng}$ interferon (IFN)- $\beta$-luc plasmid, $100 \mathrm{ng} \beta$-gal-expression plasmid, $500 \mathrm{ng}$ each immune gene expression plasmid and 1,000 ng individual ZIKV gene-encoding plasmid were transfected using the PEI reagent in the ratio of 1:2 (DNA:PEI). At $24 \mathrm{~h}$ posttransfection, transfected cells were lysed with lysis buffer supplemented with the protease inhibitor cocktail. After incubation on ice for $10 \mathrm{~min}$, the lysed cells were cleared at $15,000 \times g, 4^{\circ} \mathrm{C}$, for $15 \mathrm{~min}$. Twenty-five microliters of the sample supernatants were mixed with $25 \mu \mathrm{l}$ assay substrate and either the luciferase assay or Beta-Glo assay was performed according to the manufacturer's instructions. Firefly luciferase activities of the samples were first normalized to the internal control ( $\beta$-gal), and then the fold changes over the empty vector control were calculated. Paired two-tailed Student's t-test was performed. $p$-value $<0.05$ was considered statistically significant.

Table 2. Synthesized fragment for IRF3-5D substitution (Solid underline: mutations; amino acid substitutions are indicated below).

\begin{tabular}{|c|c|}
\hline Name & Sequence \\
\hline \multirow{5}{*}{ IRF3-5D } & GССТССТСССТGGAGAATACTGTGGACCTGCACATTGATAACGACCA \\
\hline & $S \rightarrow D$ \\
\hline & CССАCTCGATCTCGACGATGACCAGTACAAGGCCTACCTGCAGGAC \\
\hline & $\mathrm{S} \rightarrow \mathrm{D} \quad \mathrm{TS} \rightarrow \mathrm{DD}$ \\
\hline & TTGGTGGAGGGCATGGATTTCCAGGGCCCTGGGGAGAGCTGA \\
\hline
\end{tabular}


A

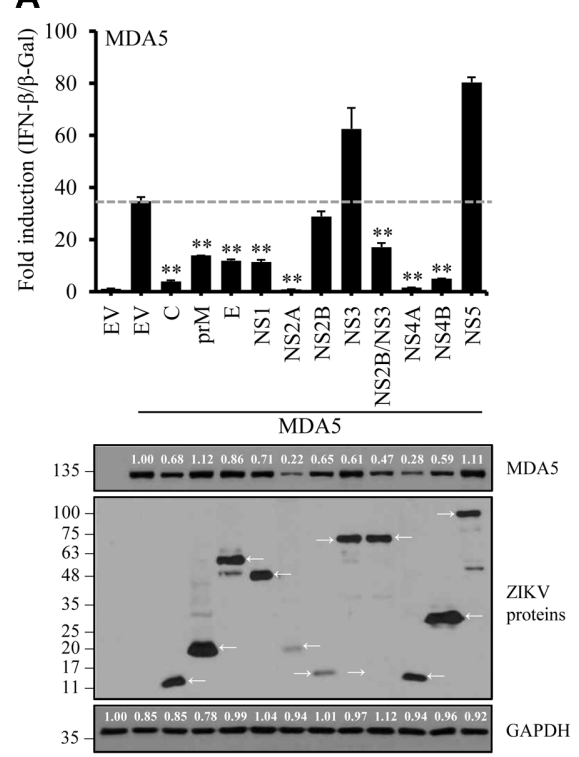

B

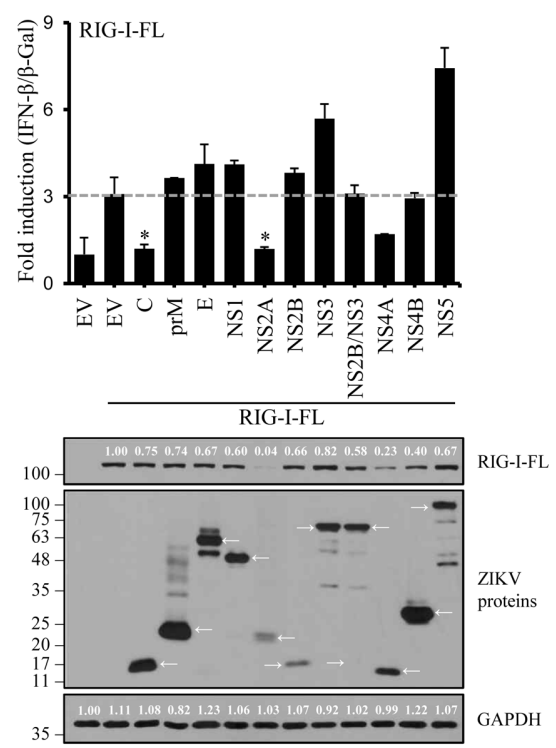

C

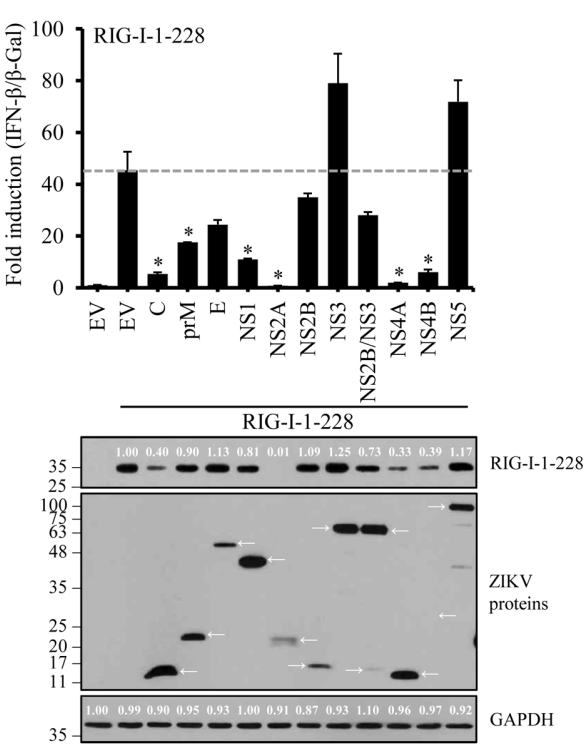

Fig. 1. ZIKV-encoded proteins suppressed the cytoplasmic RNA sensors, MDA5 and RIG-I.

HEK293T cells were co-transfected with plasmids encoding individual ZIKV protein, IFN- $\beta$-luc, $\beta$-Gal, and MDA5 (A), RIG-I-FL (B), and RIG-I-1228 (C). At $24 \mathrm{~h}$ post-transfection (hpt), the cells were lysed and used for luciferase assay (top panel). Protein expression was analyzed by western blotting (bottom panel) with anti-HA antibody (for MDA5, RIG-I, and RIG-I-1-228), anti-FLAG antibody (for ZIKV proteins), and anti-GAPDH antibody (for human GAPDH). A representative data of two independent experiments is shown. Statistical significance was determined by Student's two-tailed $t$-test ${ }^{* *} p<0.01,{ }^{*} p<0.05$. The sizes of the proteins are indicated on the left $(\mathrm{kDa})$. Arrows indicate the expected protein bands.

\section{Results}

ZIKV-Encoded C, NS2A and NS4A Strongly Inhibit Both MDA5- and RIG-I-Induced Activation of the IFN- $\beta$ Promoter Activities

To screen out the potential targets of individual ZIKV genes that affect IFN- $\beta$ production, we first tested MDA5 and RIG-I as they are cytoplasmic dsRNA sensors (Fig. 1). MDA5, full-length RIG-I (RIG-I-FL), or constitutively active RIG-I-1-228 encoding plasmids was co-transfected into HEK293T cells together with expression plasmids of each individual ZIKV gene and IFN- $\beta$-luc. Transfected cells were assayed for luciferase activity at $24 \mathrm{~h}$ post-transfection (hpt). Results showed that ZIKV C and NS2A significantly inhibited the IFN promoter activities induced by all forms of RLRs used (MDA5, RIG-I-FL, and the RIG-I-1-228 (Fig. 1)). ZIKV prM, NS1, NS4A, and NS4B decreased IFN$\beta$ promoter activation by targeting MDA5 and RIG-I-1-228, but not RIG-I-FL (Figs. 1A and 1C vs 1B). Of note, C, NS2A, and NS4A dramatically decreased both luciferase expression driven by the IFN- $\beta$ promoter and the protein levels of RLRs. These results suggest that these ZIKV-encoded proteins are capable of suppressing induction of the IFN- $\beta$ promoter activitiy by down-regulating RNA sensors of the IFN- $\beta$ pathway.

Many ZIKV-Encoded Proteins Significantly Inhibit MAVS-, TBK1-, and IKK $\varepsilon-$ Induced Activation of the IFN- $\beta$ Promoter

Next, we attempted to identify ZIKV proteins that can inhibit IFN- $\beta$ promoter activity via the downstream players of MDA5 and RIG-I, including MAVS, TBK1, and IKKe. The MAVS-, TBK1-, or IKKE-expressing plasmids were cotransfected together with each individual ZIKV gene, and activation of the IFN- $\beta$ promoter activities was assessed by luciferase assay (Fig. 2). Interestingly, NS2A and NS4A demonstrated as strong inhibitory effects on MAVS-, TBK1-, and IKK $\varepsilon$-induced activation of the IFN- $\beta$ promoter as they did on that of RLRs. Of note, NS2A-mediated inhibition seemed to be associated with its down-regulation of protein levels of MAVS, TBK1, and IKKE (Figs. 2A-2C, respectively). NS4A also moderately reduced the protein levels of TBK1 as well (Fig. 1B). Targeting of MAVS by ZIKV C, prM, NS1, NS2B, NS2B-NS3 complex, and NS4B also led to inhibition of IFN- $\beta$ promoter activity without affecting protein expression levels of MAVS (Fig. 2A). All ZIKV proteins significantly suppressed TBK1-mediated 
A

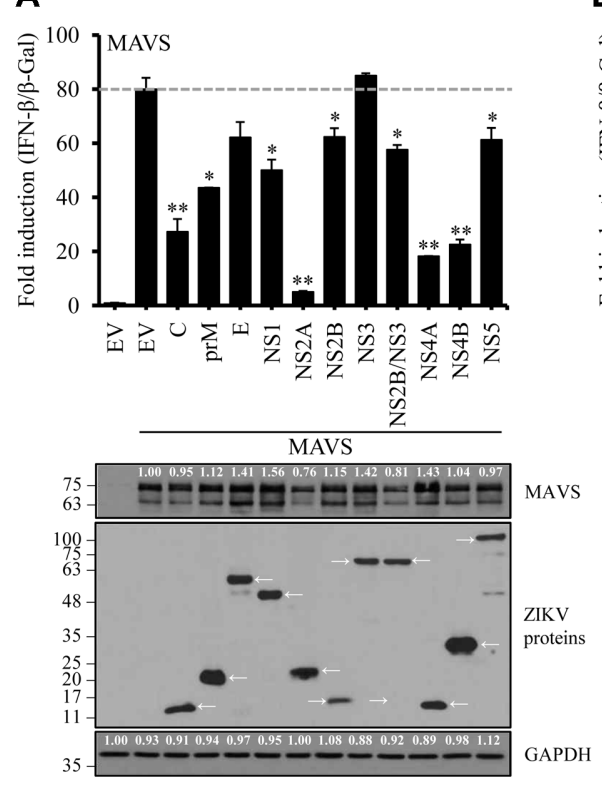

B

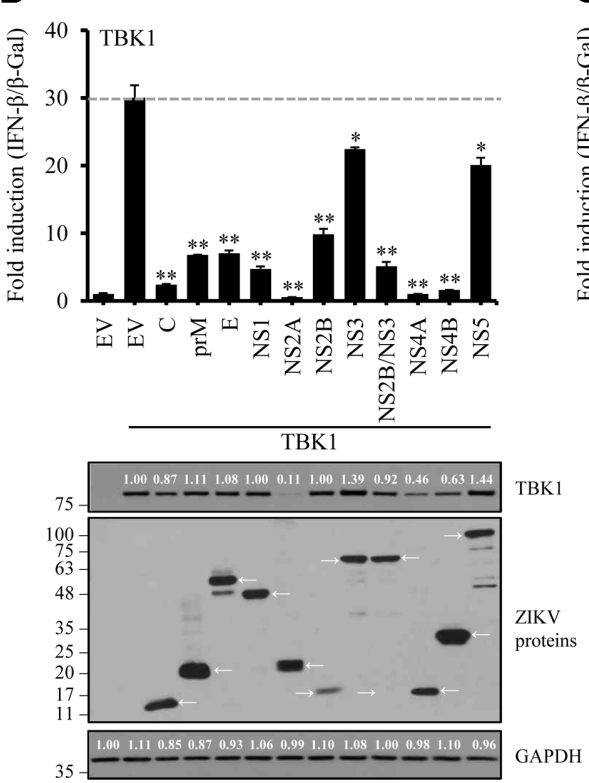

C

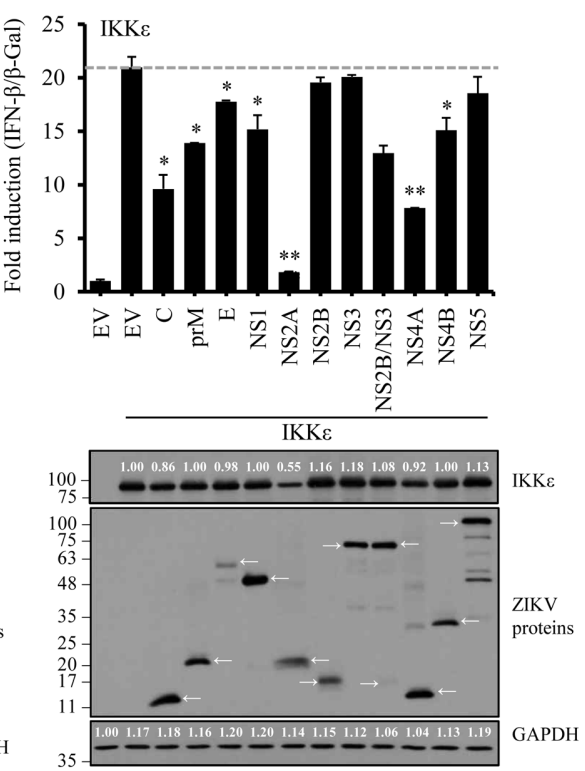

Fig. 2. ZIKV-encoded proteins antagonized activation of the IFN- $\beta$ promoter induced by MAVS, TBK1 and IKKe.

HEK293T cells were co-transfected with plasmids encoding ZIKV proteins, IFN- $\beta$-luc, $\beta$-Gal, and MAVS (A), TBK1 (B), or IKKE (C). At 24 hpt, the cells were lysed and used for the luciferase assay (top panel). Protein expression was analyzed by western blotting (bottom panel) with antiMAVS antibody or anti-HA antibody (for TBK1 and IKKE), anti-FLAG antibody (for ZIKV proteins), or anti-GAPDH antibody (for human GAPDH). The results presented are a representative of two independent experiments. Statistical significance was determined using Student's twotailed $t$-test ${ }^{* *} p<0.01,{ }^{*} p<0.05$. The size of the proteins is indicated on the left $(\mathrm{kDa})$. Arrows indicate the expected protein bands.

activation of the IFN- $\beta$ promoter; however, only NS2A substantially abated protein expression of TBK1 (roughly $89 \%$ reduction) while NS4A and NS4B decreased TBK1 protein levels by $54 \%$ and $37 \%$, respectively (Fig. $2 \mathrm{~B}$ ). Although IKK $\varepsilon$-mediated IFN- $\beta$ promoter activity was down-regulated by three structural $(\mathrm{C}, \mathrm{prM}$, and $\mathrm{E})$ and four nonstructural (NSA1, NS2A, NS4A, and NS4B) proteins, the protein levels of IKK $\varepsilon$ remained unperturbed except for NS2A, expression of which reduced IKK $\varepsilon$ protein levels by $45 \%$ (Fig. 2C).

ZIKV NS2A Is Competent to Significantly Inhibit Induction of the IFN- $\beta$ Promoter When Activated by All Forms of IRF3 (IRF3-FL, IRF3-1-290, and IRF3-5D)

IRF3 plays an important role in the expression of IFN- $\beta$ upon virus infection [27]. Phosphorylation of IRF3 by TBK1 or IKK $\varepsilon$ results in phosphorylation and homo-dimerization of IRF3, which enables its translocation into the nucleus to activate IFN- $\beta$ transcription and thus initiation of innate immune responses [28]. In this study, three IRF3-expressing constructs, including regulatory domain-deleted (IRF3-1390) and a constitutive active (IRF3-5D) and full-length IRF3 (IRF3-FL) were employed to investigate whether
ZIKV-encoded proteins are competent to inhibit activation of the IFN- $\beta$ promoter induced by any of these IRF3's (Fig. 3). Surprisingly, NS2A demonstrated inhibitory activities on IFN- $\beta$ promoter induction by all forms of IRF3's. NS4A decreased IFN- $\beta$ induction by the expression of IRF3-FL and IRF3-1-390, but not IRF3-5D (Figs. 3A and 3B). Interestingly, ZIKV E, NS1, NS2B, and NS4A significantly down-regulated IFN- $\beta$ expression only when induced by IRF3-1-390 (Fig. 3B) but not by the other two IRF3 forms (IRF3-5D and IRF3-FL).

\section{ZIKV NS2A and NS4A Inhibit MDA5-Mediated Induction of the IFN- $\beta$ Pathway in a Dose-Responsive Manner}

Varying amounts $(0,0.1,0.3$, or $1 \mu \mathrm{g})$ of plasmid expressing NS2A (Fig. 4A) or NS4A (Fig. 4B) were transfected into HEK293T cells, and luciferase assay and western blotting were performed at $24 \mathrm{hpt}$. As shown in the bottom panels of Figs. $4 \mathrm{~A}$ and $4 \mathrm{~B}, \mathrm{NS} 2 \mathrm{~A}$ or NS4A protein levels increased depending on the amounts of expression plasmids transfected. As the protein levels increased, the levels of MDA5 were decreased in a NS2A or NS4A dose-dependent manner. Accordingly, luciferase activities were decreased with inverse relationship with 
A

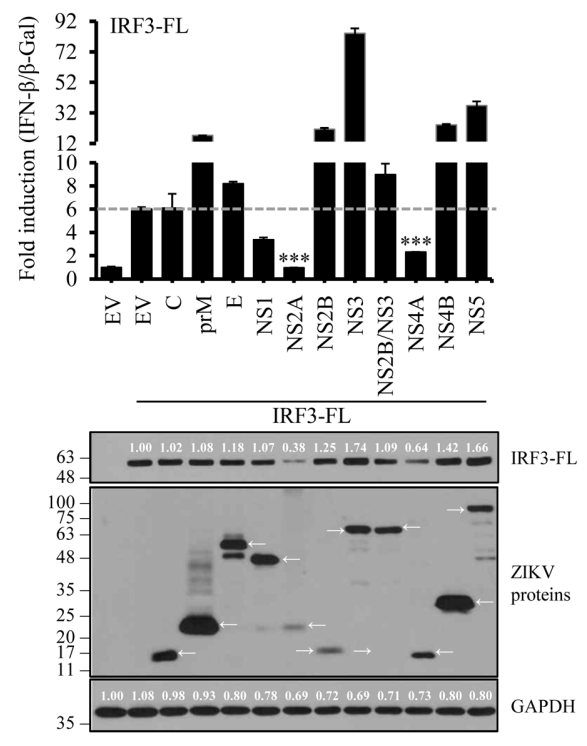

B

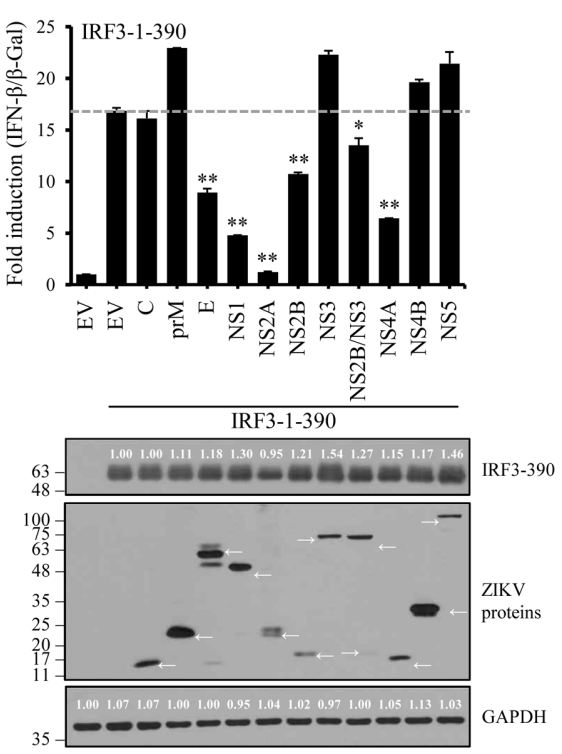

C
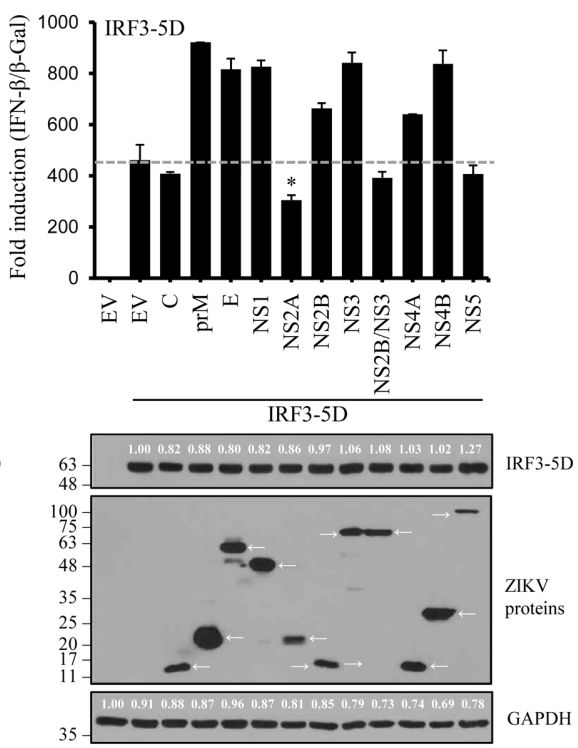

Fig. 3. ZIKV NS2A inhibits activation of the IFN- $\beta$ promoter induced by all forms of IRF3's (IRF-FL, IRF3-1-390, and IRF3-5D). (A) HEK293T cells were co-transfected with plasmids encoding ZIKV proteins, IFN- $\beta$-luc, $\beta$-Gal, and IRF3-FL (A), IRF3-390 (B), IRF3-5D (C). At 24 hpt, the cells were lysed and used for luciferase assay (top panel). Protein expression was analyzed using western blotting (bottom panel) with anti-HA antibody (for IRF3-FL, IRF3-1-390, and IRF3-5D), anti-FLAG antibody (for ZIKV proteins), and anti-GAPDH antibody (for human GAPDH). Results are representative of two independent experiments. Statistical significance was determined by the Student's two-tailed $t$-test ${ }^{* * *} p<0.001,{ }^{* *} p<0.01,{ }^{*} p<0.05$. The size of the proteins is indicated on the left $(\mathrm{kDa})$. Arrows indicate the expected protein bands.

NS2A or NS4A, clearly suggesting that ZIKV NS2A and NS4A are bona fide antagonists of MDA5-mediated activation of the IFN- $\beta$ promoter. In addition, it is likely that NS2A and NS4A activities may be responsible for the inhibition of IFN- $\beta$ promoter activity and reduction in the protein levels of MDA5.

\section{Discussion}

The aim of our study is to investigate which ZIKVencoded proteins inhibit activation of the IFN- $\beta$ promoter via the MDA5/RIG-I signaling pathway. The data presented here demonstrated that ZIKV NS2A and NS4A are the main antagonists of induction of IFN- $\beta$ by suppressing almost all the components of the MDA5/RIG-I signaling pathway with or without their effects on the protein levels of the signaling molecules involved.

The ZIKV genome encodes a single polyprotein which is proteolytically cleaved to three structural proteins (C, prM, and E) and seven non-structural proteins (NS1, NS2A, NS2B, NS3, NS4A, NS4B, and NS5). The polyprotein is cleaved by either host or viral proteases [29]. The C protein binds to the viral RNA genome to build a nucleocapsid, while prM is required for virus maturation and egression. The E protein is involved in virus-host attachment, membrane fusion, and virus entry [30]. Nonstructural proteins are mainly involved in polyprotein processing and viral genome replication and modulation of host immune responses as well. ZIKV NS1 glycoprotein, which functions as a homodimer in vivo, is an essential cofactor required for the replication of the RNA genome and viral morphogenesis. In addition, NS1 has also been shown to antagonize the innate immune response [31-33]. The alignment of the NS2A amino acid sequences among members of the Flaviviridae family revealed that the NS2A proteins are highly conserved across multiple species (> 80\%) [34]. Flavivirus NS2A proteins, an endoplasmic reticulum (ER) membrane protein, have multiple functions; viral replication, virion assembly and secretion, and inhibition of innate immunity [33, 35-40]. NS3 acts as a serine protease along with NS2B to process the polyprotein into an RNA helicase and triphosphatase [41]. NS4A is involved in the arrangement of the host membrane and the viral replication complex [42, 43]. NS4A is known to interrupt the interaction between RLRs and MAVS [44]. NS4B is involved in the formation of the viral replication 
A

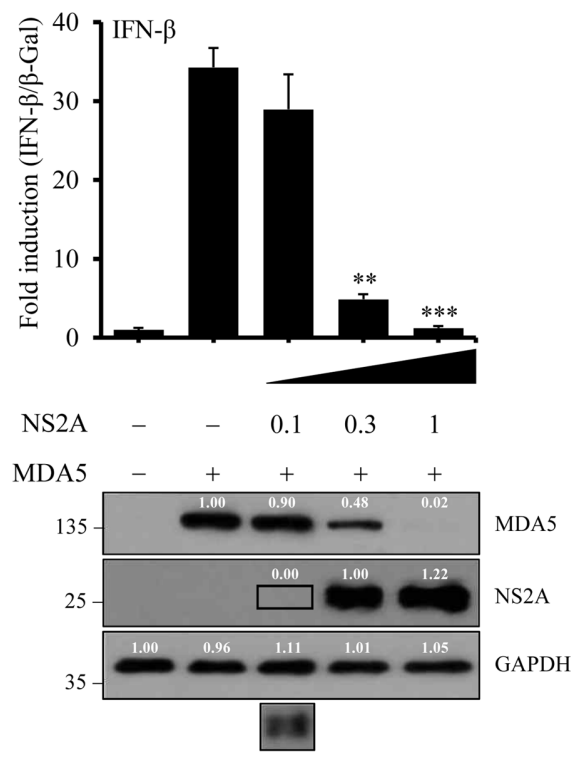

B

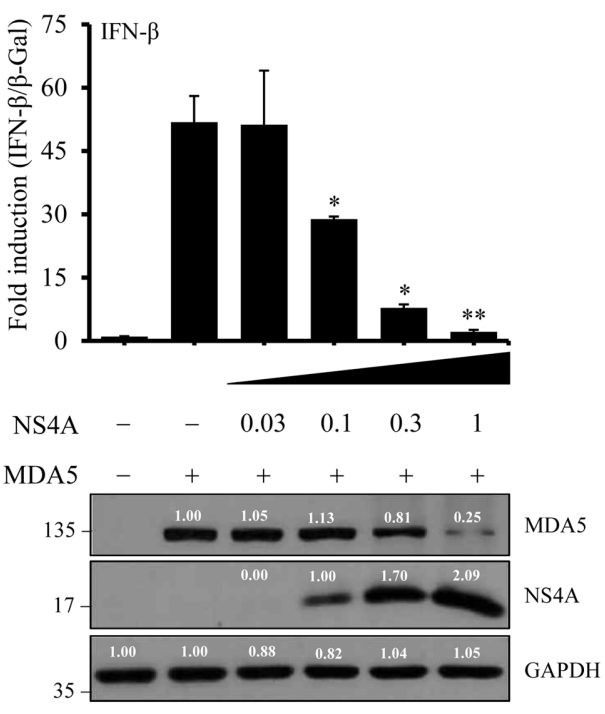

Fig. 4. ZIKV NS2A and NS4A impairs MDA5-induced IFN- $\beta$ signaling pathway in a dose-dependent manner.

Increasing amounts $(0,0.1,0.3$, or $1 \mu \mathrm{g})$ of DNA encoding ZIKV NS2A (A) ZIKV NS4A (B) were co-transfected with MDA5 (0.5 $\mu \mathrm{g})$, IFN- $\beta$-luc and $\beta$-Gal. At $24 \mathrm{hpt}$, cell lysates were prepared for luciferase assay (top panel). Protein expression was determined by western blotting (bottom panel) with anti-HA antibody (for MDA5), anti-FLAG antibody (for ZIKV proteins), and anti-GAPDH antibody (for human GAPDH). Results are representative of two independent experiments. Statistical significance was determined by the Student's two-tailed $t$-test ${ }^{* * *} p<0.001,{ }^{* *} p<0.01$, ${ }^{*} p<0.05$. The size of the proteins is indicated on the left $(\mathrm{kDa})$.

complex [45]. NS4A and NS4B inhibit the Akt-mTOR pathway to induce cellular dysregulation [46]. NS5 plays an important role in viral RNA replication as an RNAdependent RNA polymerase, and also functions as an Nterminal methyltransferase targeting $\mathrm{N}-7$ and $2^{\prime}-\mathrm{O}$ methylation of the viral RNA [47-49].

Type I IFNs are one of well-known host defense mechanisms against virus infection [50-53]. IFN secretion is initiated by the recognition of the infecting pathogen via pathogen recognition receptors (PRRs). Viral RNAs are detected by distinct RLRs, including MDA5 and RIG-I [54]. These pattern recognition receptors are key sensors for the genomes of the invading pathogens, leading to induction of the type I IFNs such as IFN- $\alpha$ and IFN- $\beta$, and proinflammatory cytokines at the early stage of virus infection. Activated RLRs cause conformational changes and then activate MAVS on the mitochondrial membrane. MAVS sequentially trigger activation of TBK1 and IKKE, resulting in the phosphorylation of IRF3. Phosphorylated IRF3 (pIRF3) dimerizes and translocates to the nucleus to induce transcription of type I IFN genes. Therefore, invading viruses should have strategies for successful infections that hinder the IFN-mediated innate immunity.
ZIKV NS2A specifically suppressed RIG-I-, MAVS-, IKK $\varepsilon-$, and TBK1-induced IFN- $\beta$ activity [33]. However, our results showed that all players of the MDA5/RIG-I signaling pathway were down-regulated by ZIKV NS2A (Figs. 1-3). ZIKV NS4A antagonizes IFN response by perturbing RIG-I and MAVS interaction [44, 55]. With the exception of IRF3-5D, ZIKV NS4A was able to downregulate all the signaling molecules involved in the MDA5/RIG-I signaling pathway (Figs. 1-3). The ZIKV NS3 and NS2B-NS3 complex are known to inhibit MAVS and mediator of IFN regulatory factor 3 activation (MITA) [56]. In contrast, our results showed no significant reduction in MAVS level by the NS3 and the NS2B-NS3 complex (Fig. 2A). Moreover, NS3 and NS2B-NS3 complex did not perturb IRF3-mediated activation of IFN responses (Fig. 3). Interestingly, NS4A demonstrated itself to be another general inhibitor of MDA5-mediated induction of IFN signaling as NS2A. That is, NS4A strongly inhibited MDA5 (Fig. 1), MAVS, TBK1, IKKe (Fig. 2) and IRF3-FL (Fig. 3). Of note, NS4A suppressed IRF3-FL (Fig. 3A) but not IRF3-5D (Fig. 3C), which is a mimic of a phosphorylated, thus active, form of IRF3. These data suggest that NS4Amediated inhibition of IRF3 may be indirect and occur 
through the inhibition of upstream IRF3 kinases (Figs. 2B and 2C): TBK1 and IKKE. Employment of TBK1- and/or IKKE-deficient cell lines would help clarify these possibilities, which is currently underway. NS4B also displays inhibitory effects on upstream signaling molecules (MDA5 (Fig. 1A), MAVS (Fig. 2A), TBK1 (Fig. 2B), and IKK $\varepsilon$ (Fig. 2C)), but not IRF3-FL (Fig. 3A). Biochemical analysis of NS4B-mediated inhibition is now in progress to determine the exact suppressive mechanisms of NS4B. On the other hand, ZIKV NS1 was found to be a 'minor' inhibitor of the IFN- $\beta$ pathway. It hampered to a lesser degree a selective set of signaling molecules involved in the signaling event: MDA5 (Fig. 1), MAVS, TBK1, and IKKE (Fig. 2), which is largely consistent with previous studies $[32,33]$. In our studies, ZIKV NS5 did not seem to inhibit any signaling molecules of IFN- $\beta$ pathway, which is in sharp contrast with a previous report [57]. Kumar et al. showed that ZIKV NS5 inhibited both IRF3 and IFN- $\beta$ promoter activities, suggesting that it may function as a ZIKV-encoded inhibitor of IRF3. However, none of three forms of IRF3's was affected by overexpression of NS5 (Fig. 3). It is interesting to note that expression of ZIKV C, NS2A, NS4A and NS4B resulted in low levels of RIG-I protein (Fig. 1B), which suggests that those ZIKV proteins may influence synthesis or direct/indirect degradation of RIG-I protein. Underlying mechanisms are now being sought. On the other hand, ZIKV prM and E protein inhibited IFN- $\beta$ production without affecting RIG-I protein levels, hinting at differential inhibitory mechanisms from those of ZIKV C, NS2A, and NS4A/4B.

Overall, our data are largely consistent with the current view of the functions of the ZIKV-encoded proteins regarding antagonism of the host innate immunity. However, our results reveal some discrepancies with some of the results of the previous studies. Those discrepancies may have stemmed from multiple reasons: 1) different human or non-human cell lines were employed in many of the studies (cell line-dependent), 2) varying sequences of ZIKV proteins were utilized (ZIKV strain-specific), 3) the size and position of tags fused to the ZIKV proteins were different (tag-dependent). Detailed and careful analysis of these issues will help resolve partial study-to-study discrepancies.

Taken together, the data presented here show that many ZIKV-encoded proteins are involved in the down-regulation of the type I IFN signaling as previously shown with minor discrepancies. More importantly, we demonstrated that ZIKV NS2A and NS4A play major roles in the inhibition of IFN- $\beta$ promoter activity by inhibiting multiple signaling mediators of the MDA5/RIG-I signaling pathway. Scrutiny of potential mechanisms of NS2A- and NS4A-mediated blockade of the type I IFN responses is currently underway. We believe that our study will add to the current understanding regarding the ZIKV-host interactions and provide clues for developing anti-ZIKV therapeutics.

\section{Acknowledgments}

This research was supported by the Basic Science Research Program of the National Research Foundation (NRF) funded by the Ministry of Education (2017R1D1A1B03035473 and 2017R1A6A1A03015876).

\section{Conflict of Interest}

The authors have no financial conflicts of interest to declare.

\section{Reference}

1. Dick GW, Kitchen SF, Haddow AJ. 1952. Zika virus. I. Isolations and serological specificity. Trans. R. Soc. Trop. Med. Hyg. 46: 509-520.

2. Cao-Lormeau VM, Blake A, Mons S, Lastere S, Roche C, Vanhomwegen J, et al. 2016. Guillain-Barre Syndrome outbreak associated with Zika virus infection in French Polynesia: a case-control study. Lancet 387: 1531-1539.

3. Chang C, Ortiz K, Ansari A, Gershwin ME. 2016. The Zika outbreak of the 21st century. J. Autoimmun. 68: 1-13.

4. Nicastri E, Castilletti C, Liuzzi G, Iannetta M, Capobianchi MR, Ippolito G. 2016. Persistent detection of Zika virus RNA in semen for six months after symptom onset in a traveller returning from Haiti to Italy, February 2016. Euro Surveill. 21(32).

5. Adams Waldorf KM, Stencel-Baerenwald JE, Kapur RP, Studholme C, Boldenow E, Vornhagen J, et al. 2016. Fetal brain lesions after subcutaneous inoculation of Zika virus in a pregnant nonhuman primate. Nat. Med. 22: 1256-1259.

6. Lazear HM, Govero J, Smith AM, Platt DJ, Fernandez E, Miner JJ, et al. 2016. A Mouse Model of Zika Virus Pathogenesis. Cell Host Microbe 19: 720-730.

7. van den Pol AN, Mao G, Yang Y, Ornaghi S, Davis JN. 2017. Zika Virus Targeting in the Developing Brain. J. Neurosci. 37: 2161-2175.

8. Wise J. 2016. Study links Zika virus to Guillain-Barre syndrome. BMJ 352: 11242.

9. Malone RW, Homan J, Callahan MV, Glasspool-Malone J, Damodaran L, Schneider Ade B, et al. 2016. Zika virus: medical countermeasure development challenges. PLoS Negl. Trop. Dis. 10(3): e0004530. 
10. Retallack H, Di Lullo E, Arias C, Knopp KA, Laurie MT, Sandoval-Espinosa C, et al. 2016. Zika virus cell tropism in the developing human brain and inhibition by azithromycin. Proc. Natl. Acad. Sci. USA 113: 14408-14413.

11. Janeway CA, Jr., Medzhitov R. 2002. Innate immune recognition. Annu. Rev. Immunol. 20: 197-216.

12. Meylan E, Tschopp J, Karin M. 2006. Intracellular pattern recognition receptors in the host response. Nature 442: 39-44.

13. Kato H, Takeuchi O, Sato S, Yoneyama M, Yamamoto M, Matsui K, et al. 2006. Differential roles of MDA5 and RIG-I helicases in the recognition of RNA viruses. Nature 441: 101105.

14. Hiscott J, Grandvaux N, Sharma S, Tenoever BR, Servant MJ, Lin R. 2003. Convergence of the NF-kappaB and interferon signaling pathways in the regulation of antiviral defense and apoptosis. Ann. NY Acad. Sci. 1010: 237-248.

15. Park MK, Cho H, Roh SW, Kim SJ, Myoung J. 2019. Cell type-specific interferon-gamma-mediated antagonism of KSHV lytic replication. Sci. Rep. 9: 2372.

16. Myoung J, Min K. 2019. Dose-dependent inhibition of melanoma differentiation-associated gene 5-mediated activation of type I interferon sresponses by methyltransferase of hepatitis E virus. J. Microbiol. Biotechnol. 29: 1137-1143.

17. Kim E, Myoung J. 2018. Hepatitis E virus papain-like cysteine protease inhibits type I interferon induction by down-regulating melanoma differentiation-associated gene 5. J. Microbiol. Biotechnol. 28: 1908-1915.

18. Baek YH, Cheon HS, Park SJ, Lloren KKS, Ahn SJ, Jeong JH, et al. 2018. Simple, rapid and sensitive portable molecular diagnosis of SFTS virus using reverse transcriptional loopmediated isothermal amplification (RT-LAMP). J. Microbiol. Biotechnol. 28: 1928-1936.

19. Phuong NH, Kwak C, Heo CK, Cho EW, Yang J, Poo H. 2018. Development and characterization of monoclonal antibodies against nucleoprotein for diagnosis of influenza A virus. J. Microbiol. Biotechnol. 28: 809-815.

20. Tong C, Chen N, Liao X, Yuan X, Sun M, Li X, et al. 2017. Continuous passaging of a recombinant $C$-strain virus in PK-15 cells selects culture-adapted variants that showed enhanced replication but failed to induce fever in rabbits. $J$. Microbiol. Biotechnol. 27: 1701-1710.

21. Xi H, Zhang K, Yin Y, Gu T, Sun Q, Shi L, et al. 2017. Fusion peptide improves stability and bioactivity of single chain antibody against rabies virus. J. Microbiol. Biotechnol. 27: 718-724.

22. Zhao K, Duan X, Hao L, Wang X, Wang Y. 2017. Immune effect of newcastle disease virus DNA vaccine with C3d as a molecular adjuvant. J. Microbiol. Biotechnol. 27: 2060-2069.

23. Lee JY, Lee JS, Materne EC, Rajala R, Ismail AM, Seto D, et al. 2018. Bacterial RecA protein promotes adenoviral recombination during in vitro infection. mSphere 3. pii: e00105-18.

24. Lee JY, Bae S, Myoung J. 2019. Middle East respiratory syndrome coronavirus-encoded accessory proteins impair
MDA5-and TBK1-mediated activation of NF-kappaB. J. Microbiol. Biotechnol. 29: 1316-1323.

25. Lee JY, Bae S, Myoung J. 2019. Middle East respiratory syndrome coronavirus-encoded ORF8b strongly antagonizes IFN-beta promoter activation: its implication for vaccine design. J. Microbiol. 57: 803-811.

26. Kang S, Choi C, Choi I, Han KN, Rho SW, Choi J, et al. 2018. Hepatitis E virus methyltransferase inhibits type I interferon induction by targeting RIG-I. J. Microbiol. Biotechnol. 28: 1554-1562.

27. Collins SE, Noyce RS, Mossman KL. 2004. Innate cellular response to virus particle entry requires IRF3 but not virus replication. J. Virol. 78: 1706-1717.

28. Liu S, Cai X, Wu J, Cong Q, Chen X, Li T, et al. 2015. Phosphorylation of innate immune adaptor proteins MAVS, STING, and TRIF induces IRF3 activation. Science 347: aaa2630.

29. Sun G, Larsen CN, Baumgarth N, Klem EB, Scheuermann RH. 2017. Comprehensive annotation of mature peptides and genotypes for Zika virus. PLoS One 12: e0170462.

30. Mukhopadhyay S, Kuhn RJ, Rossmann MG. 2005. A structural perspective of the flavivirus life cycle. Nat. Rev. Microbiol. 3: 13-22.

31. Akey DL, Brown WC, Dutta S, Konwerski J, Jose J, Jurkiw TJ, et al. 2014. Flavivirus NS1 structures reveal surfaces for associations with membranes and the immune system. Science 343: 881-885.

32. Wu Y, Liu Q, Zhou J, Xie W, Chen C, Wang Z, et al. 2017. Zika virus evades interferon-mediated antiviral response through the co-operation of multiple nonstructural proteins in vitro. Cell Discov. 3: 17006.

33. Xia H, Luo H, Shan C, Muruato AE, Nunes BTD, Medeiros DBA, et al. 2018. An evolutionary NS1 mutation enhances Zika virus evasion of host interferon induction. Nat. Commun. 9: 414.

34. Zhang X, Xie X, Zou J, Xia H, Shan C, Chen X, et al. 2019. Genetic and biochemical characterizations of Zika virus NS2A protein. Emerg. Microbes Infect. 8: 585-602.

35. Leung JY, Pijlman GP, Kondratieva N, Hyde J, Mackenzie JM, Khromykh AA. 2008. Role of nonstructural protein NS2A in flavivirus assembly. J. Virol. 82: 4731-4741.

36. Liu WJ, Chen HB, Khromykh AA. 2003. Molecular and functional analyses of Kunjin virus infectious cDNA clones demonstrate the essential roles for NS2A in virus assembly and for a nonconservative residue in NS3 in RNA replication. J. Virol. 77: 7804-7813.

37. Marquez-Jurado S, Nogales A, Avila-Perez G, Iborra FJ, Martinez-Sobrido L, Almazan F. 2018. An alanine-to-valine substitution in the residue 175 of Zika virus NS2A protein affects viral RNA synthesis and attenuates the virus in vivo. Viruses 10(10). pii: E547 
38. Munoz-Jordan JL, Sanchez-Burgos GG, Laurent-Rolle M, Garcia-Sastre A. 2003. Inhibition of interferon signaling by dengue virus. Proc. Natl. Acad. Sci. USA 100: 14333-14338.

39. Tu YC, Yu CY, Liang JJ, Lin E, Liao CL, Lin YL. 2012. Blocking double-stranded RNA-activated protein kinase PKR by Japanese encephalitis virus nonstructural protein 2A. J. Virol. 86: 10347-10358.

40. Xie X, Zou J, Puttikhunt C, Yuan Z, Shi PY. 2015. Two distinct sets of NS2A molecules are responsible for dengue virus RNA synthesis and virion assembly. J. Virol. 89: 12981313.

41. Wang CC, Huang ZS, Chiang PL, Chen CT, Wu HN. 2009. Analysis of the nucleoside triphosphatase, RNA triphosphatase, and unwinding activities of the helicase domain of dengue virus NS3 protein. FEBS Lett. 583: 691-696.

42. Miller S, Kastner S, Krijnse-Locker J, Buhler S, Bartenschlager R. 2007. The non-structural protein $4 \mathrm{~A}$ of dengue virus is an integral membrane protein inducing membrane alterations in a 2K-regulated manner. J. Biol. Chem. 282: 8873-8882.

43. Ambrose RL, Mackenzie JM. 2011. A conserved peptide in West Nile virus NS4A protein contributes to proteolytic processing and is essential for replication. J. Virol. 85: 1127411282.

44. Ma J, Ketkar H, Geng T, Lo E, Wang L, Xi J, et al. 2018. Zika virus non-structural protein $4 \mathrm{~A}$ blocks the RLR-MAVS signaling. Front Microbiol. 9: 1350.

45. Li XD, Ye HQ, Deng CL, Liu SQ, Zhang HL, Shang BD, et al. 2015. Genetic interaction between NS4A and NS4B for replication of Japanese encephalitis virus. J. Gen. Virol. 96: 1264-1275.

46. Liang Q, Luo Z, Zeng J, Chen W, Foo SS, Lee SA, et al. 2016. Zika virus NS4A and NS4B proteins deregulate Akt-mTOR signaling in human fetal neural stem cells to inhibit neurogenesis and induce autophagy. Cell Stem Cell 19: 663671.
47. Egloff MP, Benarroch D, Selisko B, Romette JL, Canard B. 2002. An RNA cap (nucleoside-2'-O-)-methyltransferase in the flavivirus RNA polymerase NS5: crystal structure and functional characterization. EMBO J. 21: 2757-2768.

48. Issur M, Geiss BJ, Bougie I, Picard-Jean F, Despins S, Mayette J, et al. 2009. The flavivirus NS5 protein is a true RNA guanylyltransferase that catalyzes a two-step reaction to form the RNA cap structure. RNA 15: 2340-2350.

49. Dong H, Fink K, Zust R, Lim SP, Qin CF, Shi PY. 2014. Flavivirus RNA methylation. J. Gen. Virol. 95: 763-778.

50. Ivashkiv LB, Donlin LT. 2014. Regulation of type I interferon responses. Nat. Rev. Immunol. 14: 36-49.

51. Taniguchi T, Takaoka A. 2002. The interferon-alpha/beta system in antiviral responses: a multimodal machinery of gene regulation by the IRF family of transcription factors. Curr. Opin. Immunol. 14: 111-116.

52. Kang S, Myoung J. 2017. Host innate immunity against hepatitis E virus and viral evasion mechanisms. J. Microbiol. Biotechnol. 27: 1727-1735.

53. Kang S, Myoung J. 2017. Primary lymphocyte infection models for KSHV and its putative tumorigenesis mechanisms in B cell lymphomas. J. Microbiol. 55: 319-329.

54. Brisse M, Ly H. 2019. Comparative structure and function analysis of the RIG-I-Like receptors: RIG-I and MDA5. Front Immunol. 10: 1586.

55. Hu Y, Dong X, He Z, Wu Y, Zhang S, Lin J, et al. 2019. Zika virus antagonizes interferon response in patients and disrupts RIG-I-MAVS interaction through its CARD-TM domains. Cell Biosci. 9: 46.

56. Li W, Li N, Dai S, Hou G, Guo K, Chen X, et al. 2019. Zika virus circumvents host innate immunity by targeting the adaptor proteins MAVS and MITA. FASEB J. 33: 9929-9944.

57. Kumar A, Hou S, Airo AM, Limonta D, Mancinelli V, Branton W, et al. 2016. Zika virus inhibits type-I interferon production and downstream signaling. EMBO Rep. 17: 17661775. 